\title{
Cardioprotective effects of rat adipose-derived stem cells differ under normoxic/physioxic conditions and are associated with paracrine factor secretion
}

\author{
YUANYUAN LIN ${ }^{1}$, XUEWEN LI $^{1}$, CHUNHUI FAN $^{2}$, FAN YANG $^{1}$, DAJIE HAO ${ }^{1}$, WENJIA GE ${ }^{3}$ and YUPING GAO ${ }^{1}$ \\ Departments of ${ }^{1}$ Cardiology, ${ }^{2}$ Information and ${ }^{3}$ Science Research and Education, \\ Shanxi Bethune Hospital, Taiyuan, Shanxi 030032, P.R. China
}

Received June 20, 2019; Accepted February 13, 2020

DOI: $10.3892 / \mathrm{ijmm} .2020 .4524$

\begin{abstract}
Adipose tissue-derived stem cells (ASCs) are beneficial for myocardial regeneration. The physiological oxygen content of human organs is estimated to range between 1 and $11 \%$. However, in the majority of previous in vitro studies with cultured ASCs, the $\mathrm{O}_{2}$ concentration was artificially set to $21 \%$. The present study aimed to compare the protective effects of rat ASCs on neonatal rat ventricular myocytes (NRVMs) under normoxic $\left(21 \% \mathrm{O}_{2}\right)$ and physioxic $\left(5 \% \mathrm{O}_{2}\right)$ conditions. Rat NRVMs cultured under normoxia or physioxia were treated with $\mathrm{H}_{2} \mathrm{O}_{2}$ or left untreated, and further co-cultured with ASCs in $21 \%$ or $5 \% \mathrm{O}_{2}$. The apoptosis of NRVMs was evaluated by Annexin $\mathrm{V}$ staining and quantitating the protein levels of Bcl-2 and Bax by western blotting. The oxidative stress of NRVMs was determined by a glutathione/oxidized glutathione assay kit. The concentrations of secreted vascular endothelium growth factor (VEGF), insulin like growth factor-1 (IGF-1) and basic fibroblast growth factor (bFGF) in the culture medium were quantified by enzyme-linked immunosorbent assay. Under both normoxia and physioxia, co-culture with ASCs protected $\mathrm{H}_{2} \mathrm{O}_{2}$-exposed NRVMs from apoptosis and significantly alleviated the oxidative stress in NRVMs. The protective effects of ASCs were associated with increased secretion of VEGF, IGF-1 and bFGF. ASCs cultured in 5\% $\mathrm{O}_{2}$ exhibited certain cardioprotective effects against $\mathrm{H}_{2} \mathrm{O}_{2}$ stress. The results of the present study suggested that $\mathrm{O}_{2}$ concentrations influenced the cardioprotective effects of ASCs. VEGF, IGF-1 and bFGF may serve a role in the myocardial regeneration mediated by transplanted ASCs.
\end{abstract}

Correspondence to: Dr Yuping Gao, Department of Cardiology, Shanxi Bethune Hospital, 99 Longcheng Street, Xiaodian, Taiyuan, Shanxi 030032, P.R. China

E-mail: gaodoct@126.com

Key words: adipose-derived stem cells, cardiomyocytes, paracrine effect

\section{Introduction}

Myocardial infarction (MI) is a leading cause of death in the world; annually, $27 \%$ of people who experience an MI die in the US (1). Current pharmaceutical agents or medical devices can rescue the dying cardiomyocytes to some extent; however, there are no effective therapeutic means that can be used to directly repair the necrotic myocardium (2). Increasing evidence suggests that adult stem cells can be applied to accelerate myocardial regeneration and improve cardiac function, including skeletal myoblasts (3) and bone marrow stem cells (4), but the difficulties in acquisition and low numbers of stem cells limit their clinical application. In 2001, Zuk et al (5) reported that a fibroblast-like population of cells from the human adipose tissue differentiated in vitro into multilineage cells in the presence of lineage-specific induction factors, and hypothesized that human adipose tissue may represent an alternative stem cell source to bone marrow-derived mesenchymal stem cells. Subsequent studies have confirmed that adipose-derived stem cells (ASCs) exist and have the capacity of self-renewal and differentiation into multiple types of cells $(6,7)$. The population of ASCs represents a promising cell source for tissue regeneration due to their easy accessibility.

Previous studies have demonstrated that ASCs improve ventricular thickness and cardiacfunction of infracted rat hearts. For example, Wang et al (8) and Schenke-Layland et al (9) have demonstrated the therapeutic efficacy of ASCs on infarcted rat heart by ligation of the left anterior descending artery. In another study, using a mouse model of myocardial infarct, Yang et al (10) revealed that both human ASCs and ASC-conditioned medium significantly reduced the myocardial infarct size and improved cardiac function. One recent clinical study reported that autologous ASCs were feasible and scalable for the treatment of ischemic cardiomyopathy (11). Increasing preclinical and clinical evidence indicates that ASC-mediated myocardial regeneration may be due to the paracrine effects of ASCs, such as anti-apoptosis, anti-cardiac remodeling and promotion of angiogenesis.

ASCs can secrete cytokines such as vascular endothelial growth factor (VEGF), transforming growth factor (TGF), hepatocyte growth factor (HGF), basic fibroblast growth factor (bFGF), placenta growth factor (PGF), granulocyte 
macrophage colony stimulating factor (GM-CSF), insulin like growth factor-1 (IGF-1) and angiopoietin (Ang) $(12,13)$. Suga et al (14) have studied the paracrine mechanism of ASCs by isolation and culturing of ASCs from transgenic mice that express luciferase and green fluorescent protein. By injecting these cells into the inguinal fat pads of wild-type mice to observe the fate of transplanted ASCs, the effects of ASC transplantation and cellular events after transplantation, they reported that ASCs exerted an angiogenic effect by activating endothelial cells through secreted cytokines HGF and VEGF in both in vitro cultured cells and in vivo mice hearts (14). As an important angiogenic factor, VEGF can promote cell migration during the process of angiogenesis (15). IGF-1 can promote proliferation and prevent apoptosis (16), and bFGF serves a significant role in promoting cell proliferation and differentiation (17). The roles of VEGF, IGF-1 and bFGF have been extensively studied in stem cell transplantation. For instance, ASC treatment in aged rats can improve aging-related erectile dysfunction partially through the secretion of IGF-1, bFGF and VEGF (18).

The physiological $\mathrm{O}_{2}$ content of human organs is estimated to range between 1 and $11 \%$ (19). However, in the majority of the in vitro studies concerning the paracrine effects of cultured ASCs, the $\mathrm{O}_{2}$ level was artificially set as $21 \%$ (irrespective of $5 \%$ $\mathrm{CO}_{2}$ supplementation), corresponding to the air oxygen content rather than the unique tissue normoxia, which was referred to as 'physioxia' by Carreau et al (19). It is estimated that the oxygen content of venous blood and ventricular myocytes in the human body is $\sim 5 \%$ (19). ASCs reside in anatomical sites that are relatively oxygen deficient, and hypoxia may provide signals conducive to the maintenance of definitive ASC properties (20). Since oxygen is a crucial component of the stem cell niches that influence cell proliferation and cell-fate commitment (21), elucidating the mechanisms on the cardioprotective effects of ASCs under physioxic $\left(5 \% \mathrm{O}_{2}\right)$ conditions rather than the artificial normoxia $\left(21 \% \mathrm{O}_{2}\right)$ is essential, as it may more accurately reflect the bona fide molecular and cellular changes in the body.

Therefore, the aim of the present study was to compare the protective effects of rat ASCs on neonatal rat ventricular myocytes (NRVMs) under normoxia and physioxia. In addition, several secreted soluble factors involved in the paracrine effects of ASCs were evaluated to determine the cardioprotective effects of ASCs.

\section{Materials and methods}

Animals. A total of 10 adult Sprague-Dawley (SD) rats (100-120 g, male) (22) and 12 neonatal SD rats (1-3 days old) were obtained from the Animal Experiment Department at Shanxi Medical University (Jinzhong, China). The rats were housed in a controlled environment $\left(20-22^{\circ} \mathrm{C}, 12 \mathrm{~h} \mathrm{light/dark}\right.$ cycle and 50\% relative humidity) and had ad libitum access to food and water. All experiments involving the use of animals were approved by the Animal Experimentation Ethics Committee of Shanxi Medical University.

Isolation and culture of rat ASCs and NRVMs. The isolation and culture of ASCs were performed as previously described (23). Briefly, adult SD rats were anesthetized with sodium pentobarbital $(30 \mathrm{mg} / \mathrm{kg}$ ) by intraperitoneal injection, and their inguinal fat pads were removed, followed by cervical dislocation. Fat pads were rinsed several times with D-Hanks' solution. Blood vessels and fibrous tissues were excised under a dissecting microscope and discarded. The remaining adipose tissues were minced into $\sim 1 \mathrm{~mm}^{3}$ sections, digested with $0.2 \%$ type II collagenase (cat. no. 17101-015; Gibco; Thermo Fisher Scientific, Inc.) for $10 \mathrm{~min}$ and agitated for $1 \mathrm{~h}$ at $37^{\circ} \mathrm{C}$. Following centrifugation at $168 \mathrm{xg}$ for $10 \mathrm{~min}$ at $4^{\circ} \mathrm{C}$, the cell pellet was resuspended in the ASC culture medium (Dulbecco's Modified Eagle's Medium supplemented with $15 \%$ fetal bovine serum, $100 \mathrm{U} / \mathrm{ml}$ penicillin and $100 \mu \mathrm{g} / \mathrm{ml}$ streptomycin; all from Thermo Fisher Scientific, Inc.). The ASCs were transferred into culture flasks and incubated with $5 \% \mathrm{CO}_{2}$ at $37^{\circ} \mathrm{C}$. The medium was changed $24 \mathrm{~h}$ after plating and replaced every 2 days. ASCs at passage 3 were used in the following experiments.

The isolation and culture of cardiomyocytes were conducted as previously described (24). Cardiac tissue was derived from the hearts of neonatal SD rats aged 1-3 days. Animals were sacrificed with carbon dioxide (10-30\% volume displacement $/ \mathrm{min}$ ) followed by cervical dislocation. The hearts were removed and washed in ice-cold PBS. The left ventricles of the rats were collected and digested with type II collagenase. The NRVMs were plated at a density of $1 \times 10^{5}$ cells $/ \mathrm{ml}$ in cell culture medium (Dulbecco's Modified Eagle's Medium supplemented with $10 \%$ fetal bovine serum, $100 \mathrm{U} / \mathrm{ml}$ penicillin and $100 \mu \mathrm{g} / \mathrm{ml}$ streptomycin) containing $0.1 \mathrm{mM}$ bromodeoxyuridine (cat. no. B9285; Sigma-Aldrich; Merck $\mathrm{KGaA}$ ) to inhibit the proliferation of non-cardiomyocytes. The NRVMs exhibited spontaneous contraction and synchronous beat frequency was examined under microscope. The purity of cardiomyocytes was examined under a microscope by immunocytochemical identification of specific sarcomeric $\alpha$-actin. NRVMs in the primary culture were used in the following experiments, and the cells were cultured in the incubator with $5 \% \mathrm{CO}_{2}$ and $21 \% \mathrm{O}_{2}$ or $5 \% \mathrm{CO}_{2}$ and $5 \% \mathrm{O}_{2}$ at $37^{\circ} \mathrm{C}$.

Co-culture of NRVMs and ASCs. NRVMs were inoculated into $10 \mathrm{~cm}$ culture dishes and cultured for $48 \mathrm{~h}$ under normoxic $\left(21 \% \mathrm{O}_{2}\right.$, normoxia group) or physioxic $\left(5 \% \mathrm{O}_{2}\right.$, physioxia group) conditions. NRVMs were treated with $0,50,100,200$, 400 and $800 \mu \mathrm{M} \mathrm{H}_{2} \mathrm{O}_{2}$ for $3 \mathrm{~h}$ at $37^{\circ} \mathrm{C}(25)$. For co-culture, NRVMs were transferred into 6-well plates, and ASCs were inoculated into the chambers nested in the 6-well plates. ASCs and NRVMs were co-cultured at a ratio of 1:5 in ASC culture medium. NRVMs in the normoxia group were co-cultured with ASCs overnight under normoxia and further cultured under normoxia for $24 \mathrm{~h}$. To facilitate cell adherence, NRVMs in the physioxia group were first co-cultured with ASCs overnight under normoxia, and subsequently cultured under physioxia for $24 \mathrm{~h}$.

Immunofluorescence staining. NRVMs were cultured on glass slides, fixed with $4 \%$ paraformaldehyde for $10 \mathrm{~min}$ at $37^{\circ} \mathrm{C}$ and washed with PBS. The cells were incubated with an anti-sarcomeric $\alpha$-actinin antibody (1:200 cat. no. ab137346; Abcam) in PBS supplemented with $1 \%$ bovine serum albumin (cat. no. 0332; Amresco, Inc.) overnight at $4^{\circ} \mathrm{C}$. Following washing with PBS, the cells were incubated with 
a FITC-conjugated goat anti-rabbit IgG secondary antibody (1:1,000; cat. no. ab6717; Abcam) for $30 \mathrm{~min}$ at $37^{\circ} \mathrm{C}$. Finally, the cells were stained with $10 \mu \mathrm{g} / \mathrm{ml}$ DAPI for $10 \mathrm{~min}$ at room temperature and observed under a Nikon Ni-U fluorescence microscope (magnification, x200) (Nikon Corporation, Japan), and three fields were analyzed per sample.

Flow cytometry. The phenotypic markers of ASCs (CD29 and CD44) (26) and the negative markers of ASCs (CD31, CD106, CD184, CD34 and CD45) were detected by flow cytometry to identify rat ASCs. Following washing with PBS containing 3\% FBS, ASCs $\left(200 \mu \mathrm{l} ; 3 \times 10^{6}\right.$ cells $\left./ \mathrm{ml}\right)$ were incubated with $2 \mu \mathrm{l}$ phycoerythrin (PE)-labeled anti-rat CD29 (cat. no. sc-9970; Santa Cruz Biotechnology, Inc.) or PE-labeled anti-rat CD44 (cat. no. sc-53069; Santa Cruz Biotechnology, Inc.) at $4^{\circ} \mathrm{C}$ for $1 \mathrm{~h}$ away from light. After washing with PBS supplemented with $3 \%$ FBS twice, ASCs were centrifuged at $168 \times \mathrm{g}$ for $5 \mathrm{~min}$ at $4^{\circ} \mathrm{C}$ and resuspended in PBS. A corresponding isotype control antibody (cat. no. sc-2855; Santa Cruz Biotechnology, Inc.) was used to set up the staining background. Cells were analyzed using a FACSCalibur flow cytometer (BD Biosciences), and the results were analyzed with the CellQuest acquisition software (BD Biosciences).

The apoptotic rate of NRVMs was assessed by the Annexin V-FITC Apoptosis Detection kit (Beyotime Institute of Biotechnology) according to the manufacturer's protocol (25). Briefly, NRVMs were washed with ice-cold PBS and resuspended at a density of $1 \times 10^{6}$ cells $/ \mathrm{ml}$ in $100 \mu \mathrm{l}$ binding buffer. The cells were incubated with $5 \mu \mathrm{l}$ Annexin V-FITC solution in the dark for $15 \mathrm{~min}$ at room temperature. Following washing with PBS, the cells were resuspended in $100 \mu \mathrm{l}$ PBS, mixed with $5 \mu \mathrm{l}$ propidium iodide solution and analyzed by flow cytometry as aforementioned.

ELISA. To estimate the concentrations of secreted VEGF, IGF-1 and bFGF, the conditioned medium of untreated and $\mathrm{H}_{2} \mathrm{O}_{2}$-treated NRVMs cultured alone or co-cultured with ASCs under normoxia or physioxia was collected and subjected to ELISA. ELISA was performed using the VEGF Rat ELISA kit (Beijing Jiamay Biotech Co., Ltd.), Mouse/Rat IGF-I Quantikine ELISA kit (R\&D Systems, Inc.) and Mouse/Rat FGF basic Quantikine ELISA kit (R\&D Systems, Inc.) according to the manufacturers' protocols.

Myocardial glutathione (GSH) measurement. Following co-culture, NRVMs were minced and broken up with PBS. After centrifugation at $2,054 \times \mathrm{g}$ for $10 \mathrm{~min}$ at $4^{\circ} \mathrm{C}$, the supernatant was collected to measure the levels of total GSH and oxidized glutathione (GSSG) using the total Glutathione/Oxidized Glutathione Assay kit (cat. no. A061-1; Nanjing Jiancheng Bioengineering Institute) according to the manufacturer's instructions. The GSH/GSSG ratio was calculated to determine the levels of oxidative stress in NRVMs.

Western blotting. NRVMs were collected, washed with PBS, lysed in 1\% Cell Lysis Buffer (Abcam) and centrifuged for $10 \mathrm{~min}$ at 24,149 $\mathrm{x}$ g. The protein concentration was determined using a BCA Protein Assay kit (Thermo Fisher Scientific, Inc.). A total of $30 \mu \mathrm{g}$ protein/lane was loaded onto $12 \%$ SDS-PAGE and transferred to a PVDF membrane. Following blocking with $5 \%$ non-fat dry milk, the membrane was incubated with a rat anti-Bcl-2 monoclonal antibody (1:500; cat. no. sc-7382; Santa Cruz Biotechnology, Inc.), mouse anti-Bax monoclonal antibody (1:500; cat. no. sc-493; Santa Cruz Biotechnology, Inc.) or rabbit anti-rat $\beta$-actin polyclonal antibody $(1: 1,000$; cat. no. ab8227; Abcam) overnight at $4^{\circ} \mathrm{C}$. The membranes were then washed with TBS $+0.5 \%$ Tween- 20 and incubated with horseradish peroxidase-conjugated secondary antibodies (goat anti-rat/mouse/rabbit; 1:4,000; cat. no. A0208; Beyotime Institute of Biotechnology). The ECL kit (Thermo Fisher Scientific, Inc.) was used to visualize the bands. The intensities of the protein bands were quantified using a Gel Doc XR ${ }^{+}$ Photo-Image System with Quantity One software version 4.6 (Bio-Rad Laboratories, Inc.). The western blot experiments were repeated three times.

Statistical analysis. SPSS 17.0 (SPSS, Inc.) was used for statistical analysis. Data are presented as the mean \pm standard deviation. Comparisons between two groups were performed using Student's t-test, whereas one-way ANOVA with Bonferroni post hoc test was used for comparisons among multiple groups. $\mathrm{P}<0.05$ was considered to indicate a statistically significant difference.

\section{Results}

Characterization of rat ASCs. Rat ASCs were round with floating lipid vesicles in the culture medium when initially inoculated into the culture dishes (Fig. 1A). The cells gradually extended and exhibited a short fusiform shape after 3 days. On day 7-8 after inoculation, the ASCs began to proliferate and exhibited a fibroblast-like appearance. After the primary ASCs were cultured for 3-5 passages, they proliferated with adherence and formed a monolayer during further culture for 3-4 days. Then, ASCs displayed uniform spindle shape and 'whirlpool-like' growth at different magnification after four days (Fig. 1A). Flow cytometry analysis revealed that the ASCs were positive for the surface expression of CD29 and CD44 (Fig. 1B) and negative for that of CD31, CD106, CD184, CD34 and CD45 (Fig. 2).

Characterization of NRVMs. The isolated NRVMs were validated by characterizing their morphology, unique property of spontaneous contraction and muscle tissue-specific expression of sarcomeric- $\alpha$ actinin. The NRVMs were adherent at $24 \mathrm{~h}$ post-plating (Fig. 1C). The NRVMs exhibited spontaneous contraction with synchronous beat frequency of 80-150 beats per minute. The cells were long rod-, polygonal or spindle-shaped with concentric or radial growth. Immunofluorescence staining of sarcomeric $\alpha$-actinin confirmed that the purity of the cultured NRVMs was $>90 \%$ (Fig. 1D).

Co-culture with ASCs protects $\mathrm{H}_{2} \mathrm{O}_{2}$-treated $\mathrm{NRVMs}$ from apoptosis under normoxia or physioxia. In order to determine whether ASCs may exert the protective effects on damaged NRVMs, treatment with $\mathrm{H}_{2} \mathrm{O}_{2}$ was used to induce injury in NRVMs. First, the toxicity of $\mathrm{H}_{2} \mathrm{O}_{2}$ was tested at various concentrations $(50,100,200,400$ and $800 \mu \mathrm{M})$. The results demonstrated that treatment with $>100 \mu \mathrm{M} \mathrm{H}_{2} \mathrm{O}_{2}$ for 


\section{A}

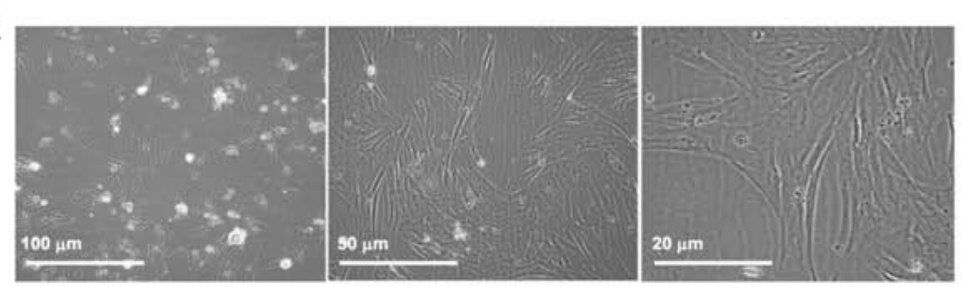

C



B
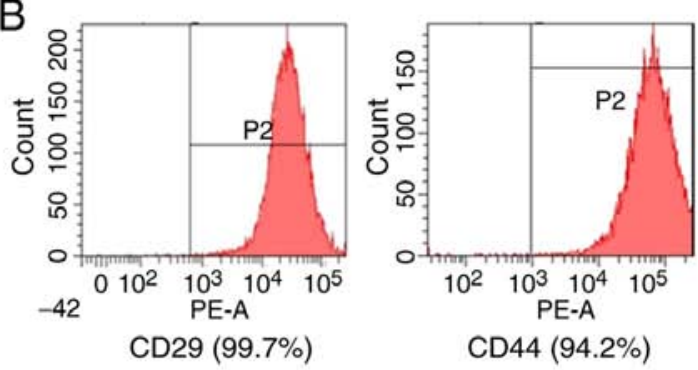

$\mathrm{D}$

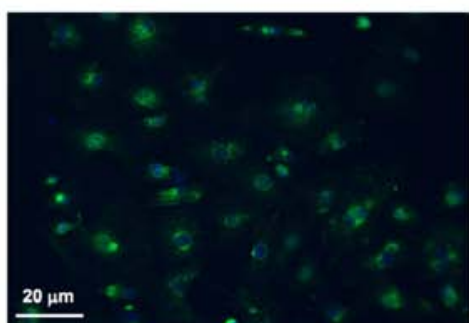

Figure 1. Characterization of rat ASCs and NRVMs. (A) Representative images demonstrating the morphology of cultured primary rat ASCs after four days under a light microscope. Images captured under different magnifications are presented. Scale bar (left to right), 100, 50 and $20 \mu \mathrm{m}$. (B) Representative histogram profiles of the surface expression of CD29 and CD44 on rat ASCs determined by flow cytometry. (C) Representative images demonstrating the morphology of cultured primary rat NRVMs under a light microscope. Images captured under different magnifications are presented. Scale bar (left to right), 100,50 and $20 \mu \mathrm{m}$. (D) Representative image of the immunofluorescence staining of sarcomeric $\alpha$-actinin (green) in primary rat NRVMs. The nuclei were stained with DAPI (blue). Scale bar, $20 \mu \mathrm{m}$. ASCs, adipose tissue-derived stem cells; NRVMs, neonatal rat ventricular myocytes.
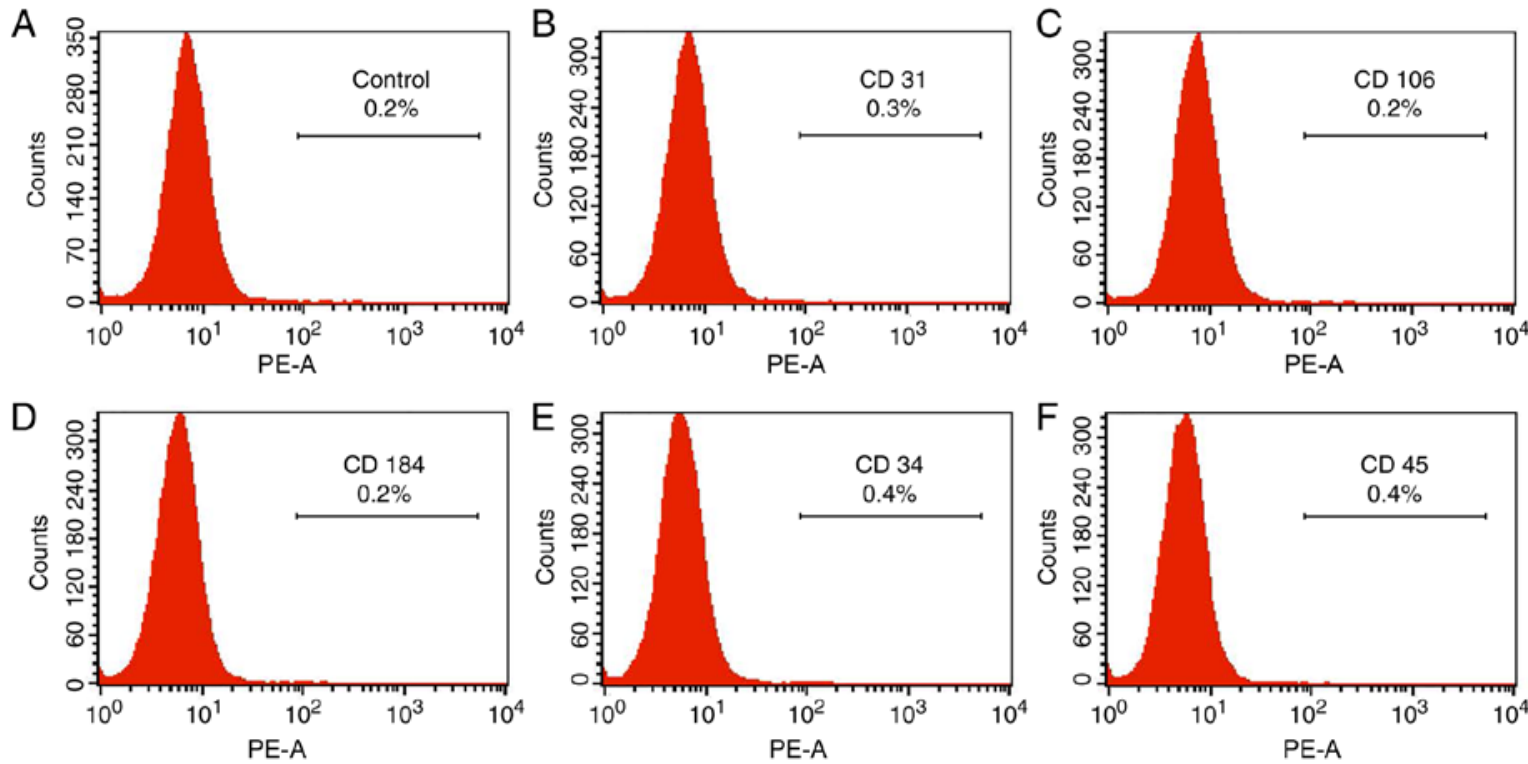

Figure 2. Immunophenotyping of the ASCs. Representative flow cytometry histogram profiles demonstrated the surface expression of (A) control, (B) CD31, (C) CD106, (D) CD184, (E) CD34 and (F) CD45 on rat ASCs. ASCs, adipose tissue-derived stem cells.

$4 \mathrm{~h}$ resulted in the death of the majority of NRVMs (data not shown). Therefore, $100 \mu \mathrm{M} \mathrm{H}_{2} \mathrm{O}_{2}$ was selected to induce damage in NRVMs in subsequent experiments.

A NRVM/ASC co-culturing system was established to evaluate the protective roles of ASCs on $100 \mu \mathrm{M} \mathrm{H}_{2} \mathrm{O}_{2}$-injured NRVMs. The morphology analysis of the co-cultured NRVMs revealed that co-culture with ASCs facilitated the recovery of damaged NRVMs; the viability of NRVMs was increased in the co-culture with ASCs group compared with that of the $\mathrm{H}_{2} \mathrm{O}_{2}$-injured NRVMs. This effect was more evident under physioxia compared with normoxia (Fig. 3A).
The apoptotic rate of NRVMs following co-culture was detected by flow cytometry. The apoptotic rate of NRVM following $100 \mu \mathrm{M} \mathrm{H}_{2} \mathrm{O}_{2}$ treatment was significantly increased under normoxia (17.54\%) and physioxia (14.31\%) compared with that of the untreated control $(1.05 \%)(\mathrm{P}<0.05$; Fig. 3B). The extent to which NRVMs were damaged by $\mathrm{H}_{2} \mathrm{O}_{2}$ (as reflected by the apoptotic rate) did not differ significantly under normoxia or physioxia conditions. When co-cultured with ASCs under normoxia, the apoptotic rate of NRVMs was significantly decreased compared with that of NRVMs cultured alone $(4.77 \%$ vs. $21.42 \%$, respectively; $\mathrm{P}<0.05$; 
A Non-treated NRVMs+ASCs (in $21 \% \mathrm{O}_{2}$ )

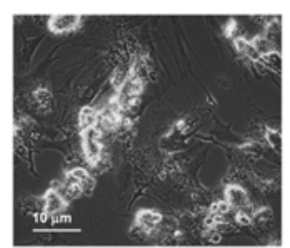

$\mathrm{H}_{2} \mathrm{O}_{2}$-treated NRVMs+ASCs (in $21 \% \mathrm{O}_{2}$ )

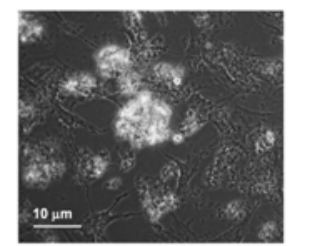

$\mathrm{H}_{2} \mathrm{O}_{2}$-treated NRVMs+ASCs (in $5 \% \mathrm{O}_{2}$ )

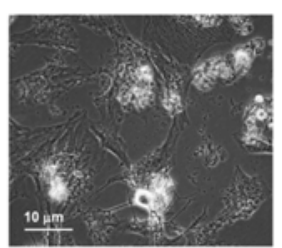

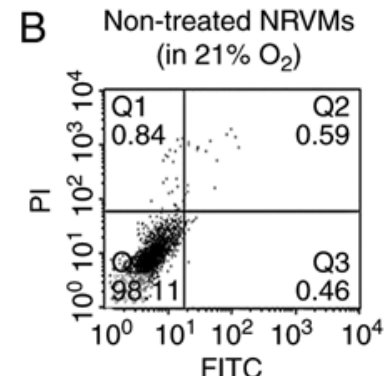

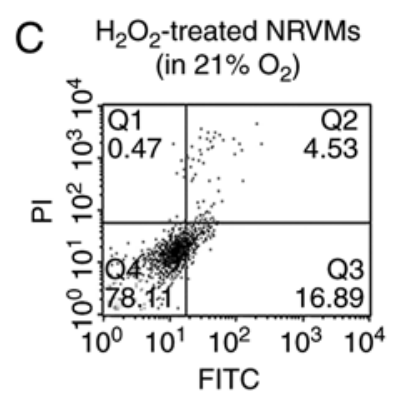

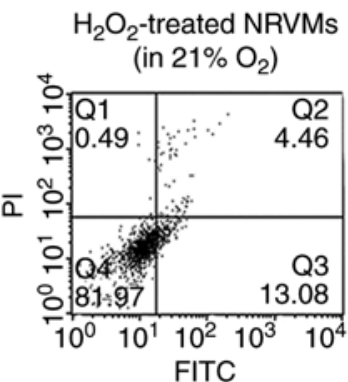

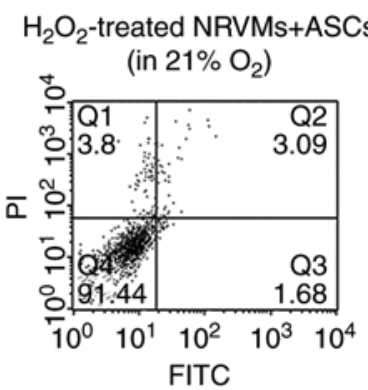

$\square \mathrm{HON}$-treated NRVMs (in $21 \% \mathrm{O}_{2}$ ) $\otimes \mathrm{H}_{2} \mathrm{O}_{2}$-treated NRVMs (in 21\% $\mathrm{O}_{2}$ ) $\boxminus \mathrm{H}_{2} \mathrm{O}_{2}$-treated NRVMs (in $5 \% \mathrm{O}_{2}$ )
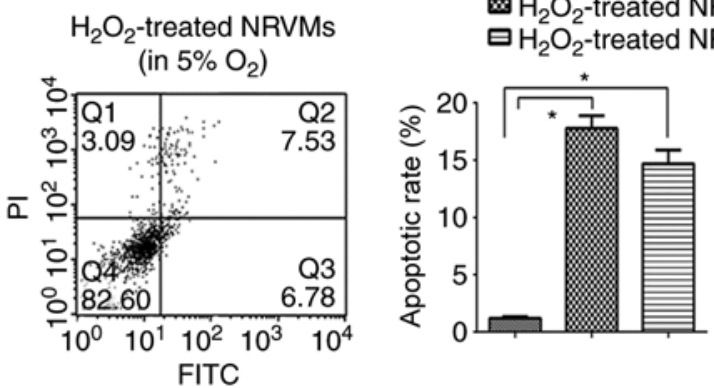

$\mathrm{H}_{2} \mathrm{O}_{2}$-treated NRVMs (in $21 \% \mathrm{O}_{2}$ )

$\otimes \mathrm{H}_{2} \mathrm{O}_{2}$-treated NRVMs + ASCs (in $21 \% \mathrm{O}_{2}$ )

Figure 3. Co-cultured rat ASCs protect $\mathrm{H}_{2} \mathrm{O}_{2}$-injured NRVMs from apoptosis. (A) Representative images of the morphological changes in non-treated or $\mathrm{H}_{2} \mathrm{O}_{2}$-treated NRVMs co-cultured with rat ASCs under normoxia $\left(21 \% \mathrm{O}_{2}\right)$ or physioxia $\left(5 \% \mathrm{O}_{2}\right)$. Scale bar, $10 \mu \mathrm{m}$. (B and C) Apoptotic rates of NRVMs non-treated or $\mathrm{H}_{2} \mathrm{O}_{2}$-treated NRVMs under normoxia or physioxia (B) alone or (C) co-cultured with rat ASCs were detected by flow cytometry. The apoptotic rate was defined as the percentage of FITC-Annexin V positive population (UR + LR). UL, upper left; UR, upper right; LL, lower left; LR, lower right; ASCs, adipose tissue-derived stem cells; NRVMs, neonatal rat ventricular myocytes.

Fig. 3C), suggesting that ASCs exerted a cardioprotective effect in normoxia. The apoptotic rate of NRVMs was decreased when NRVMs were co-cultured with ASCs under physioxia (data not shown).

The protein levels of the pro-apoptotic protein Bax and the anti-apoptotic protein $\mathrm{Bcl}-2$ were determined by western blotting to evaluate the apoptotic status of NRVMs co-cultured with ASCs. In $\mathrm{H}_{2} \mathrm{O}_{2}$-injured NRVMs, the level of Bcl-2 protein was significantly decreased, but the level of Bax protein exhibited no significant difference in normoxia compared with untreated cells (Fig. 4A). Under physioxia, the level of Bax protein was further increased, whereas the level of Bcl-2 protein was further decreased in $\mathrm{H}_{2} \mathrm{O}_{2}$-injured NRVMs compared with untreated cells under normoxia (Fig. 4A). In the $\mathrm{H}_{2} \mathrm{O}_{2}$-injured NRVMs co-cultured with ASCs, the protein expression of Bax was downregulated, and the protein expression of $\mathrm{Bcl}-2$ was upregulated in normoxia compared with $\mathrm{H}_{2} \mathrm{O}_{2}$-injured NRVMs cultured alone (Fig. 4B). Of note, in $\mathrm{H}_{2} \mathrm{O}_{2}$-injured NRVMs co-cultured ASCs, the apoptotic rate of NRVMs appeared to be more inhibited under physioxia compared with normoxia, as evidenced by the reduced expression of Bax protein and increased expression of $\mathrm{Bcl}-2$ protein in NRVMs co-cultured with ASCs in $5 \% \mathrm{O}_{2}$ (Fig. 4C).
Co-culture with ASCs under physioxia significantly alleviates the oxidative stress in $\mathrm{H}_{2} \mathrm{O}_{2}$-injured $\mathrm{NRVMs}$. It has been observed that oxidative stress increases in the injured myocardium (27). The GSH/GSSG ratio, a measure of overall cellular oxidative stress (28), was used in the present study to evaluate the degree of myocardial injury in NRVMs cultured under various conditions. As presented in Fig. 5A, exposure to $100 \mu \mathrm{M} \mathrm{H}_{2} \mathrm{O}_{2}$ significantly increased the levels of oxidative stress in NRVMs cultured alone or co-cultured with ASCs. Under normoxia, the GSH/GSSG ratio was lower in $\mathrm{H}_{2} \mathrm{O}_{2}$ stimulated NRVMs compared with that in untreated NRVMs $(\mathrm{P}<0.05)$; compared with the $\mathrm{H}_{2} \mathrm{O}_{2}$-injured NRVMs cultured alone, the GSH/GSSG ratio was not increased in the NRVMs co-cultured with ASCs (Fig. 5A). However, under physioxia, the GSH/GSSG ratio was significantly increased in $\mathrm{H}_{2} \mathrm{O}_{2}$-treated NRVMs co-cultured with ASCs compared with those cultured alone $(\mathrm{P}<0.05$; Fig. $5 \mathrm{~B})$. Taken together, these results suggested that co-culture with ASCs in physioxia, but not normoxia, alleviated the oxidative stress in damaged NRVMs.

Co-culture of $\mathrm{H}_{2} \mathrm{O}_{2}$-injured NRVMs with ASCs increases the secretion of VEGF, IGF-1 and bFGF under normoxia or physioxia. To explore whether the paracrine mechanism of 

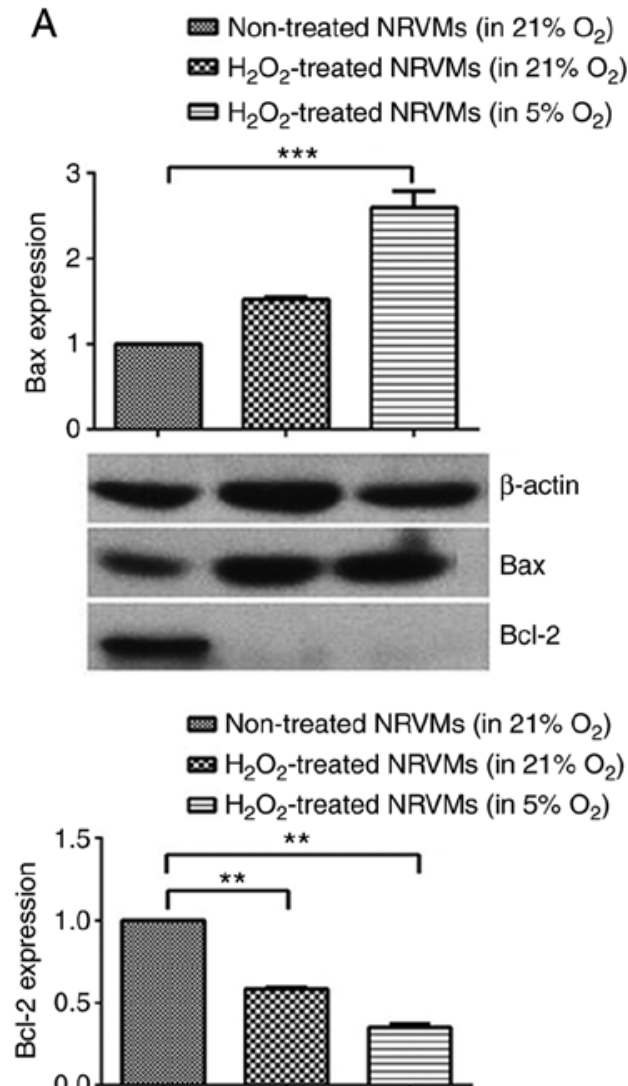

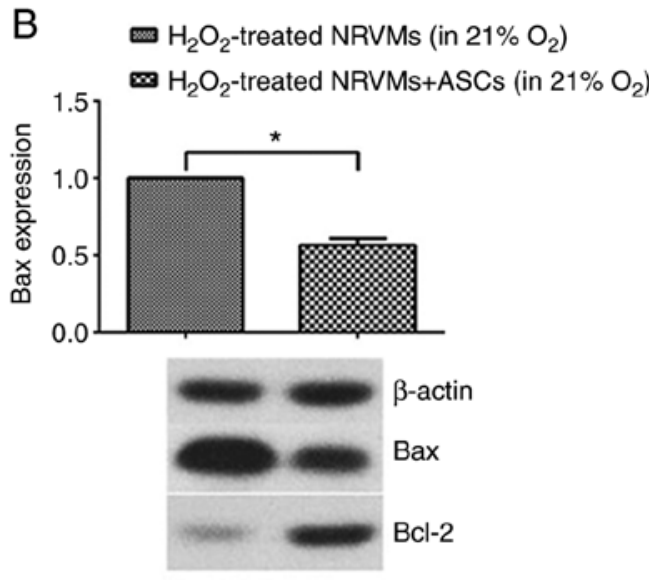

$\mathrm{H}_{2} \mathrm{O}_{2}$-treated NRVMs (in $21 \% \mathrm{O}_{2}$ )

$\approx \mathrm{H}_{2} \mathrm{O}_{2}$-treated NRVMs+ASCs (in $21 \% \mathrm{O}_{2}$ )

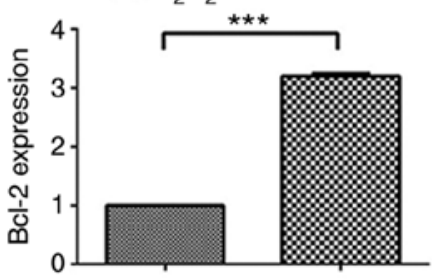

C $\quad \begin{aligned} & \mathrm{H}_{2} \mathrm{O}_{2} \text {-treated NRVMs+ASCs (in } 5 \% \mathrm{O}_{2} \text { ) } \\ & \mathrm{H}_{2} \mathrm{O}_{2} \text {-treated NRVMs+ASCs (in } 21 \% \mathrm{O}_{2} \text { ) }\end{aligned}$
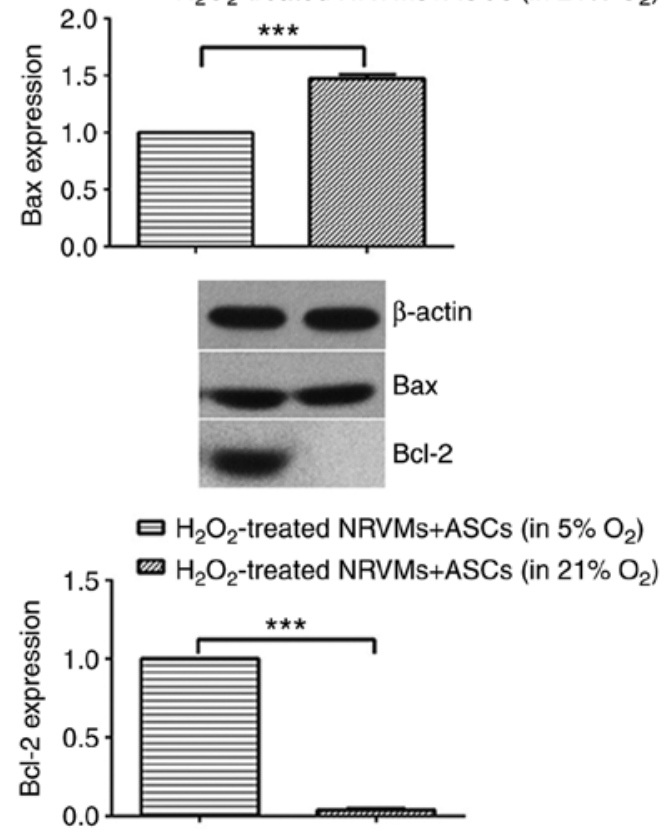

Figure 4. The expression levels of apoptosis-related proteins Bax and Bcl-2 in NRVMs were determined by western blotting. (A) Expression levels of Bax and Bcl-2 proteins in untreated and $\mathrm{H}_{2} \mathrm{O}_{2}$-treated NRVMs under normoxia $\left(21 \% \mathrm{O}_{2}\right)$ and physioxia $\left(5 \% \mathrm{O}_{2}\right)$. (B) Comparison between the expression levels of Bax and Bcl-2 proteins in $\mathrm{H}_{2} \mathrm{O}_{2}$-treated NRVMs cultured alone or co-cultured with ASCs under normoxic conditions (C) Comparison between the expression levels of Bax and Bcl-2 proteins in $\mathrm{H}_{2} \mathrm{O}_{2}$-treated NRVMs co-cultured with ASCs under normoxia and physioxia. $\beta$-actin was used as loading control. Data are representative of three independent experiments. ${ }^{*} \mathrm{P}<0.05,{ }^{* *} \mathrm{P}<0.01$ and ${ }^{* * * *} \mathrm{P}<0.001$. ASCs, adipose tissue-derived stem cells; NRVMs, neonatal rat ventricular myocytes.

ASCs contributed to their cardioprotective effect, the levels of soluble factors VEGF, IGF-1 and bFGF in the co-culture medium were measured. Under normoxia, the level of VEGF in the culture supernatant was decreased after the NRVMs (cultured alone or co-cultured with ASCs) were treated with $\mathrm{H}_{2} \mathrm{O}_{2}$ compared with untreated cells. Compared with the 

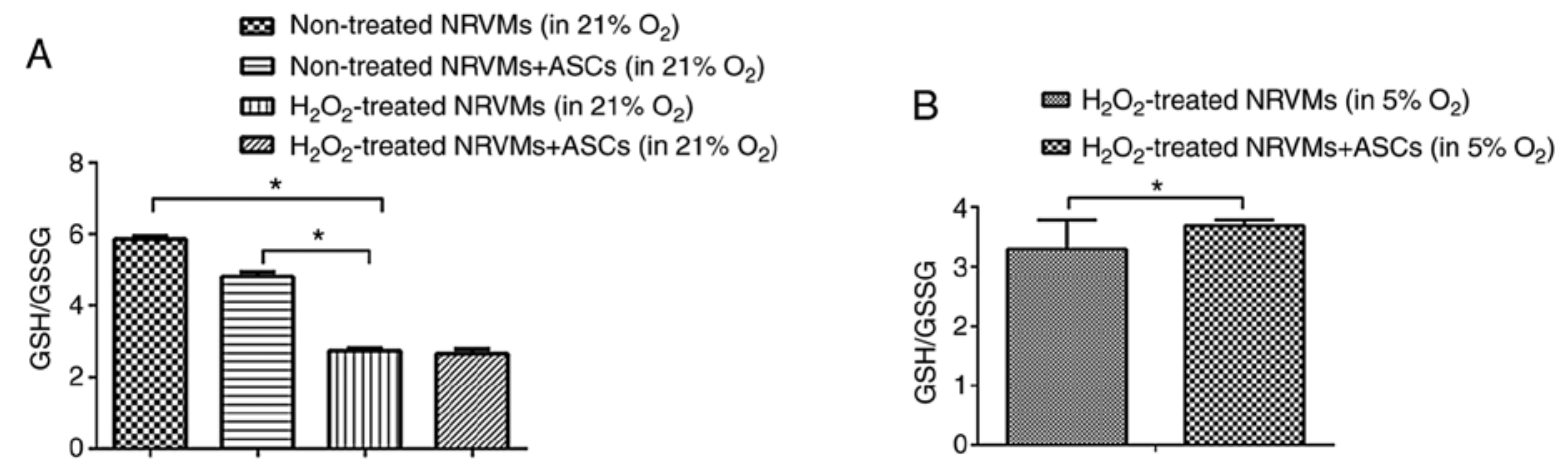

Figure 5. Co-culture with ASCs under physioxic conditions alleviates oxidative stress in $\mathrm{H}_{2} \mathrm{O}_{2}$-injured NRVMs. (A and B) The GSH/GSSG ratio in untreated and $\mathrm{H}_{2} \mathrm{O}_{2}$-treated NRVMs under normoxia $\left(21 \% \mathrm{O}_{2}\right)$ and physioxia $\left(5 \% \mathrm{O}_{2}\right.$ ). (A) Exposure to $100 \mu \mathrm{M} \mathrm{H}_{2} \mathrm{O}_{2}$ significantly decreased the GSH/GSSG ratio in NRVMs cultured alone or co-cultured with ASCs. (B) Under physioxia and $\mathrm{H}_{2} \mathrm{O}_{2}$ exposure-mediated NRVMs injury, the GSH/GSSG ratio was significantly increased when NRVMs were co-cultured with ASCs. "P<0.05. ASCs, adipose tissue-derived stem cells; NRVMs, neonatal rat ventricular myocytes; GSH, glutathione; GSSG, oxidized glutathione.
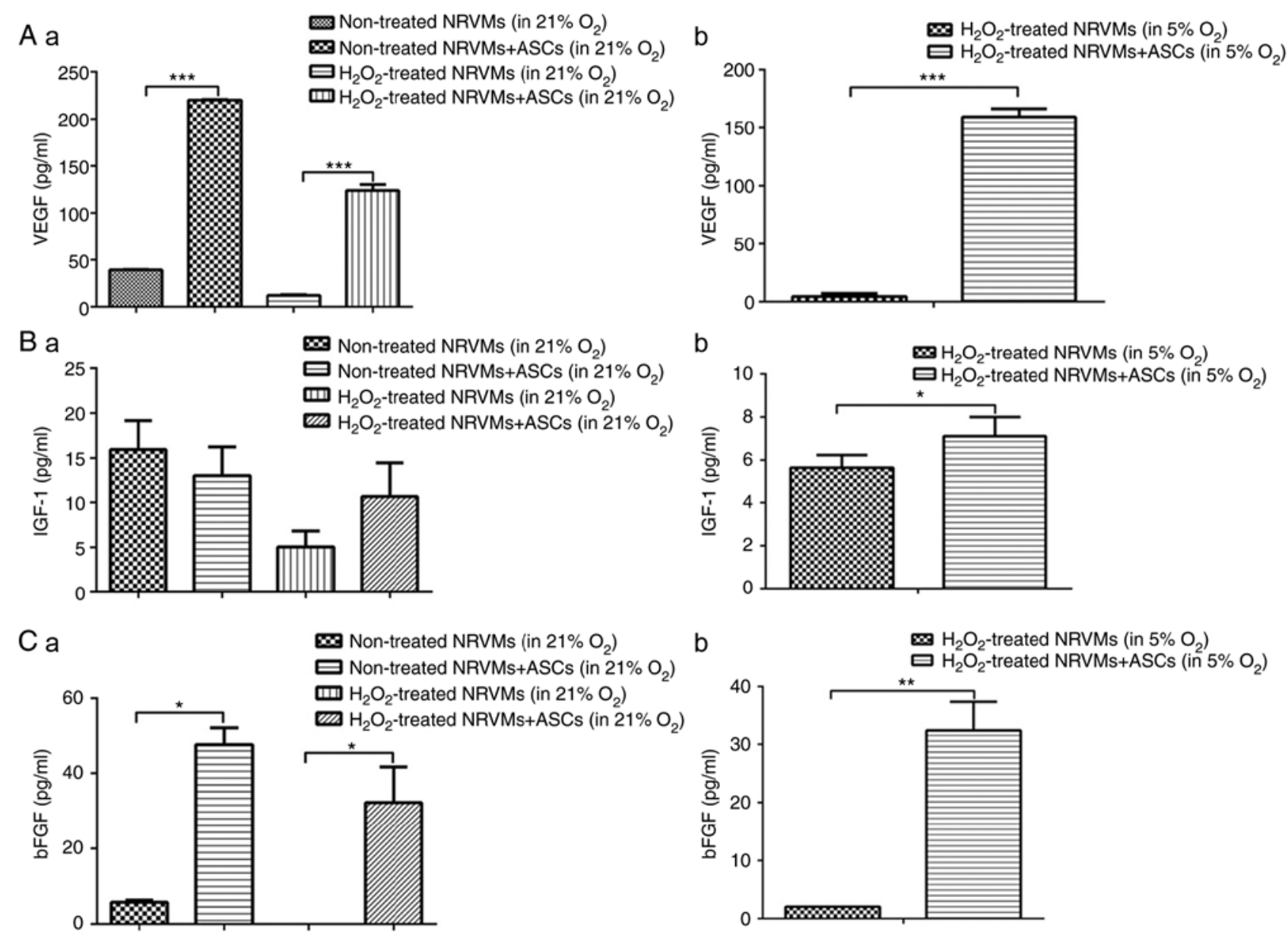

Figure 6. Co-culture of $\mathrm{H}_{2} \mathrm{O}_{2}$-injured NRVMs with ASCs increases the secretion of VEGF, IGF-1 and bFGF under normoxia or physioxia. (A) The levels of VEGF in the culture supernatant of untreated and $\mathrm{H}_{2} \mathrm{O}_{2}$-treated NRVMs under normoxia $\left(21 \% \mathrm{O}_{2}\right)$ and physioxia $\left(5 \% \mathrm{O}_{2}\right)$. (A-a) The levels of secreted VEGF in different groups under normoxia. (A-b) Under physioxia, ASCs co-cultured with injured NRVMs increased the secretion of VEGF. (B) The levels of IGF-1 in the culture supernatant of untreated and $\mathrm{H}_{2} \mathrm{O}_{2}$-treated NRVMs under normoxia and physioxia. (B-a) The levels of secreted IGF-1 in different groups under normoxia. (B-b) Under physioxia, ASCs co-cultured with injured NRVMs increased the secretion of IGF-1. (C) The levels of bFGF in culture supernatant of untreated and $\mathrm{H}_{2} \mathrm{O}_{2}$-treated NRVMs under normoxia and physioxia. (C-a) The levels of bFGF in different groups under normoxia. (C-b) Under physioxia, ASCs co-cultured with injured NRVMs increased the secretion of bFGF. ${ }^{*} \mathrm{P}<0.05,{ }^{* *} \mathrm{P}<0.01$ and ${ }^{* * *} \mathrm{P}<0.001$. ASCs, adipose tissue-derived stem cells; NRVMs, neonatal rat ventricular myocytes; VEGF, vascular endothelial growth factor; IGF-1, insulin-like growth factor; bFGF, basic fibroblast growth factor.

NRVMs cultured alone, untreated or $\mathrm{H}_{2} \mathrm{O}_{2}$-injured NRVMs co-cultured with ASCs exhibited significantly increased levels of VEGF in the supernatant $(\mathrm{P}<0.001$; Fig. 6A). Under physioxia, when the $\mathrm{H}_{2} \mathrm{O}_{2}$-injured NRVMs were co-cultured with ASCs, the VEGF concentration in the medium was significantly increased compared with that in the medium 
of NRVMs cultured alone (Fig. 6A). Co-culture with ASCs slightly increased the secretion of IGF-1 in $\mathrm{H}_{2} \mathrm{O}_{2}$-injured NRVMs under normoxia compared with NRVMs cultured alone, although the difference was not significant (Fig. 6B). However, under physioxia, co-culture of injured $\mathrm{H}_{2} \mathrm{O}_{2}$-NRVMs with ASCs significantly increased IGF-1 secretion into the culture medium compared with that in NRVMs cultured alone $(\mathrm{P}<0.05$; Fig. 6B). Similarly, under normoxia, the exposure of NRVMs to $\mathrm{H}_{2} \mathrm{O}_{2}$ reduced the concentration of secreted bFGF to an undetectable level, whereas co-culture with ASCs significantly increased the level of soluble bFGF compared with NRVMs cultured alone ( $\mathrm{P}<0.05$; Fig. 6C). Consistently, when cells were cultured in $5 \% \mathrm{O}_{2}$, co-culture with ASCs increased the bFGF content in the culture supernatant of the $\mathrm{H}_{2} \mathrm{O}_{2}$-injured NRVMs compared with NRVMs cultured alone $(\mathrm{P}<0.01$; Fig. 6C). Collectively, these results indicated that the elevated levels of soluble factors VEGF, IGF-1 and bFGF in the co-culture condition may contribute to the cardioprotective effect of ASCs.

\section{Discussion}

Nearly half of patients with cardiovascular disease die from ischemic heart failure, and most of them suffer from myocardial infarction (29). The necrotic myocardium cannot be repaired or reversed, and the current treatment is limited to the approach of recovering with blood reperfusion, which does not consistently result in a favorable outcome (29). Transplantation of stem cells for myocardial repair therapy is a promising treatment method that has attracted wide attention (29). ASCs have been demonstrated to possess the ability of multiple lineage differentiation (30) with the potential to treat myocardial infarction. However, the cardioprotective effects of ASCs under physioxia, which represents the oxygen content of venous blood and ventricular myocytes, have not been investigated. The present study established an in vitro NRVM/ASC co-culture model to compare the protective effects of ASCs on $\mathrm{H}_{2} \mathrm{O}_{2}$-injured NRVMS under 21 and $5 \% \mathrm{O}_{2}$ conditions. The results demonstrated that ASCs in $5 \% \mathrm{O}_{2}$ exerted a stronger protective effect against $\mathrm{H}_{2} \mathrm{O}_{2}$-induced damage on NRVMs compared with ASCs in $21 \% \mathrm{O}_{2}$ in terms of apoptosis inhibition in NRVMs and paracrine secretion of soluble factors such as VEGF, IGF-1 and bFGF.

As ASCs are derived from the fat tissue and their acquisition induces less pain compared with bone marrow-derived mesenchymal stem cells, the clinical and translational application of ASCs has become a research hotspot, especially in the field of treating myocardial infarction $(31,32)$. A previous study has demonstrated that ASC transplantation promotes angiogenesis and improves myocardial remodeling after myocardial infarction to improve cardiac function (33). In the majority of the studies concerning the beneficial effects of in vitro cultured ASCs, the oxygen condition was either artificially set as normoxia or hypoxia $\left(<5 \% \mathrm{O}_{2}\right)$. For example, Sadat et al (34) reported that in an in vitro neonatal rat cardiomyocyte and ASC co-culture system, the anti-apoptotic effect of ASCs was observed under hypoxic conditions (a humidified atmosphere with $95 \% \mathrm{~N}_{2}$ and $5 \% \mathrm{CO}_{2}$ gas mixture). Consistent with the results of the present study, a previous study demonstrated that the ASCs markedly suppressed oxygen deprivation-induced cardiomyocyte cell death, and the oxygen content in their cell culture condition was also set as $5 \% \mathrm{O}_{2}$ (35). To the best of our knowledge, the present study is the first report directly comparing the beneficial effects of ASCs under normoxia and physioxia on ventricular myocytes following oxidative stress. The results of the present study suggested that oxygen deprivation may influence the molecular and cellular behavior of ASCs, particularly their paracrine effects.

ASCs can differentiate into mature adipocytes, myocardium and vascular endothelium under certain conditions (36). However, further understanding obtained in recent years poses a challenge to this view; a number of studies have suggested that mesenchymal stem cells cannot differentiate into cardiomyocytes and vascular cells, and their therapeutic functions may be dependent on their paracrine effects $(37,38)$. This implies that the paracrine mechanism in the therapeutic effect of ASCs serves an important role. To further clarify the paracrine mechanism of ASCs, a model of $\mathrm{H}_{2} \mathrm{O}_{2}$-induced NRVMs injury was established in the present study to evaluate the effects of ASCs on NRVM apoptosis. The results demonstrated that ASCs significantly reduced $\mathrm{H}_{2} \mathrm{O}_{2}$-induced NRVM apoptosis in normoxia. Similarly to other stem cells, ASCs secrete a variety of soluble factors such as VEGF, HGF and IGF-1, which may participate in the cardiac repair process, including the reduction of myocardial apoptosis, the promotion of angiogenesis, increasing the survival rate of transplanted cells, reducing inflammation and mobilizing endogenous cardiac stem cells $(39,40)$.

In the present study, notably increased secretion of VEGF, IGF-1 and bFGF was observed after co-culture of NRVMs and ASCs compared with that in NRVMs cultured alone, which may help explain the protective role of ASCs by paracrine secretion of VEGF, IGF-1 and bFGF. A previous study has demonstrated that ASCs promote the secretion of VEGF and HGF in a hypoxic environment, and a moderate or low amount of active oxygen can improve ASC expansion, migration and regeneration (41). The present study confirmed the protective effects of ASCs on cardiomyocytes under physioxia, for which $5 \%$ oxygen was chosen. However, whether VEGF, IGF-1 and bFGF also contributed to the activation of ASCs themselves in the co-culture system under the $5 \% \mathrm{O}_{2}$ condition needs to be further studied. The main limitation of the present study was that an ASC culture group was not used as a control; additional experimental groups and soluble factors are required to verify the cardioprotective role of ASCs by paracrine secretion. In addition, although the clinical application of ASCs appears promising, the main mechanisms of how ASCs improve cardiac function have not been fully elucidated. Further in vivo experiments with multiple animal models are warranted to provide insights on the translational use of ASCs in the clinic.

In conclusion, using an in vitro rat ASC/NRVM co-culture system, the cardioprotective effects of ASCs were evaluated under 21 and $5 \% \mathrm{O}_{2}$ conditions. The results demonstrated that oxygen concentrations influenced the cardioprotective effects of ASCs. VEGF, IGF-1 and bFGF may serve a role in the myocardial regeneration mediated by transplanted ASCs. The present study highlights the crucial roles of the paracrine effects of ASCs in treating myocardial diseases such as myocardial infarction. 


\section{Acknowledgements}

Not applicable.

\section{Funding}

This study was supported by a grant from the International Science and Technology Cooperation program of Shanxi Province (grant no. 2014081050-2).

\section{Availability of data and materials}

The datasets used and analyzed during the current study are available from the corresponding author on reasonable request.

\section{Authors' contributions}

YPG and YYL performed the experiments, collected the data and drafted the manuscript. XWL and FY performed the statistical analysis and participated in the study design. CHF, DJH and WJG contributed analysis tools, analyzed the data and participated in the drafting of the manuscript. All authors read and approved the final manuscript.

\section{Ethics approval and consent to participate}

All experiments involving the use of animals were approved by the Animal Experimentation Ethics Committee of Shanxi Medical University.

\section{Patient consent for publication}

Not applicable.

\section{Competing interests}

The authors declare that they have no competing interests.

\section{References}

1. Boateng $\mathrm{S}$ and Sanborn T: Acute myocardial infarction. Dis Mon 59: 83-96, 2013.

2. Alagarsamy KN, Yan W, Srivastava A, Desiderio V and Dhingra S: Application of injectable hydrogels for cardiac stem cell therapy and tissue engineering. Rev Cardiovasc Med 20: 221-230, 2019.

3. Povsic TJ, O'Connor CM, Henry T, Taussig A, Kereiakes DJ, Fortuin FD, Niederman A, Schatz R, Spencer R 4th, Owens D, et al: A double-blind, randomized, controlled, multicenter study to assess the safety and cardiovascular effects of skeletal myoblast implantation by catheter delivery in patients with chronic heart failure after myocardial infarction. Am Heart J 162: 654-662. e1, 2011.

4. Liu B, Duan CY, Luo CF, Ou CW, Sun K, Wu ZY, Huang H, Cheng CF, Li YP and Chen MS: Effectiveness and safety of selected bone marrow stem cells on left ventricular function in patients with acute myocardial infarction: A meta-analysis of randomized controlled trials. Int J Cardiol 177: 764-770, 2014.

5. Zuk PA, Zhu M, Mizuno H, Huang J, Futrell JW, Katz AJ, Benhaim P, Lorenz HP and Hedrick MH: Multilineage cells from human adipose tissue: Implications for cell-based therapies. Tissue Eng 7: 211-228, 2001.

6. Poglio S, De Toni-Costes F, Arnaud E, Laharrague P, Espinosa E, Casteilla L and Cousin B: Adipose tissue as a dedicated reservoir of functional mast cell progenitors. Stem Cells 28: 2065-2072, 2010 .
7. Gimble JM and Guilak F: Differentiation potential of adipose derived adult stem (ADAS) cells. Curr Top Dev Biol 58: 137-160, 2003.

8. Wang L, Deng J, Tian W, Xiang B, Yang T, Li G, Wang J, Gruwel M, Kashour T, Rendell J, et al: Adipose-derived stem cells are an effective cell candidate for treatment of heart failure: An MR imaging study of rat hearts. Am J Physiol Heart Circ Physiol 297: H1020-H1031, 2009.

9. Schenke-Layland K, Strem BM, Jordan MC, Deemedio MT, Hedrick MH, Roos KP, Fraser JK and Maclellan WR: Adipose tissue-derived cells improve cardiac function following myocardial infarction. J Surg Res 153: 217-223, 2009.

10. Yang D, Wang W, Li L, Peng Y, Chen P, Huang H, Guo Y, Xia X, Wang Y, Wang $\mathrm{H}$, et al: The relative contribution of paracine effect versus direct differentiation on adipose-derived stem cell transplantation mediated cardiac repair. PLoS One 8: e59020, 2013.

11. Henry TD, Pepine CJ, Lambert CR, Traverse JH, Schatz R, Costa M, Povsic TJ, David Anderson R, Willerson JT, Kesten S and Perin EC: The athena trials: Autologous adipose-derived regenerative cells for refractory chronic myocardial ischemia with left ventricular dysfunction. Catheter Cardiovasc Interv 89: 169-177, 2017.

12. Hsiao ST, Lokmic Z, Peshavariya H, Abberton KM, Dusting GJ, Lim SY and Dilley RJ: Hypoxic conditioning enhances the angiogenic paracrine activity of human adipose-derived stem cells. Stem Cells Dev 22: 1614-1623, 2013.

13. Rehman J, Traktuev D, Li J, Merfeld-Clauss S, Temm-Grove CJ, Bovenkerk JE, Pell CL, Johnstone BH, Considine RV and March KL: Secretion of angiogenic and antiapoptotic factors by human adipose stromal cells. Circulation 109: 1292-1298, 2004.

14. Suga H, Glotzbach JP, Sorkin M, Longaker MT and Gurtner GC: Paracrine mechanism of angiogenesis in adipose-derived stem cell transplantation. Ann Plast Surg 72: 234-241, 2014

15. Hoeben A, Landuyt B, Highley MS, Wildiers H, Van Oosterom AT and De Bruijn EA: Vascular endothelial growth factor and angiogenesis. Pharmacol Rev 56: 549-580, 2004.

16. Youssef A, Aboalola D and Han VK: The roles of insulin-like growth factors in mesenchymal stem cell niche. Stem Cells Int 2017: 9453108, 2017.

17. Kim JH, Lee MC, Seong SC, Park KH and Lee S: Enhanced proliferation and chondrogenic differentiation of human synovium-derived stem cells expanded with basic fibroblast growth factor. Tissue Eng Part A 17: 991-1002, 2011.

18. Yang J, Zhang Y, Zang G, Wang T, Yu Z, Wang S, Tang Z and Liu J: Adipose-derived stem cells improve erectile function partially through the secretion of IGF-1, bFGF, and VEGF in aged rats. Andrology 6: 498-509, 2018.

19. Carreau A, El Hafny-Rahbi B, Matejuk A, Grillon C and Kieda C: Why is the partial oxygen pressure of human tissues a crucial parameter? Small molecules and hypoxia. J Cell Mol Med 15: 1239-1253, 2011.

20. Kim JH, Park SH, Park SG, Choi JS, Xia Y and Sung JH: The pivotal role of reactive oxygen species generation in the hypoxia-induced stimulation of adipose-derived stem cells. Stem Cells Dev 20: 1753-1761, 2011.

21. Mohyeldin A, Garzón-Muvdi T and Quiñones-Hinojosa A: Oxygen in stem cell biology: A critical component of the stem cell niche. Cell Stem Cell 7: 150-161, 2010.

22. Pushpan CK, V S, G S, Rathnam P, A J and A H: Attenuation of atherosclerotic complications by modulating inflammatory responses in hypercholesterolemic rats with dietary Njavara rice bran oil. Biomed Pharmacother 83: 1387-1397, 2016.

23. Tchkonia T, Tchoukalova YD, Giorgadze N, Pirtskhalava T, Karagiannides I, Forse RA, Koo A, Stevenson M, Chinnappan D, Cartwright A, et al: Abundance of two human preadipocyte subtypes with distinct capacities for replication, adipogenesis, and apoptosis varies among fat depots. Am J Physiol Endocrinol Metab 288: E267-E277, 2005.

24. Rutering J, Ilmer M, Recio A, Coleman M, Vykoukal J and Alt E: Improved method for isolation of neonatal rat cardiomyocytes with increased yield of C-Kit+ cardiac progenitor cells. J Stem Cell Res Ther 5: 1-8, 2015.

25. Chen A, Li G, Chen L, Guo J and Liu Y: Downregulation of microRNA-100 protects $\mathrm{H}_{2} \mathrm{O}_{2}$-induced apoptosis in neonatal cardiomyocytes. Int J Clin Exp Pathol 8: 5491-5496, 2015.

26. Xu Y, Liu Z, Liu L, Zhao C, Xiong F, Zhou C, Li Y, Shan Y Peng $F$ and Zhang $C$ : Neurospheres from rat adipose-derived stem cells could be induced into functional Schwann cell-like cells in vitro. BMC Neurosci 9: 21, 2008. 
27. Waddingham MT, Sonobe T, Tsuchimochi H, Edgley AJ, Sukumaran V, Chen YC, Hansra SS, Schwenke DO, Umetani K, Aoyama K, et al: Diastolic dysfunction is initiated by cardiomyocyte impairment ahead of endothelial dysfunction due to increased oxidative stress and inflammation in an experimental prediabetes model. J Mol Cell Cardiol 137: 119-131, 2019.

28. Owen JB and Butterfiel DA: Measurement of oxidized/reduced glutathione ratio. Methods Mol Biol 648: 269-277, 2010

29. Fang Z, Yin X, Wang J, Tian N, Ao Q, Gu Y and Liu Y: Functional characterization of human umbilical cord-derived mesenchymal stem cells for treatment of systolic heart failure. Exp Ther Med 12: 3328-3332, 2016.

30. Cawthorn WP, Scheller EL and MacDougald OA: Adipose tissue stem cells meet preadipocyte commitment: Going back to the future. J Lipid Res 53: 227-246, 2012.

31. Zhu Y, Liu T, Song K, Fan X, Ma X and Cui Z: Adipose-derived stem cell: A better stem cell than BMSC. Cell Biochem Funct 26: 664-675, 2008

32. Li TS, Cheng K, Malliaras K, Smith RR, Zhang Y, Sun B, Matsushita N, Blusztajn A, Terrovitis J, Kusuoka H, et al: Direct comparison of different stem cell types and subpopulations reveals superior paracrine potency and myocardial repair efficacy with cardiosphere-derived cells. J Am Coll Cardiol 59: 942-953, 2012

33. Mazo M, Hernández S, Gavira JJ, Abizanda G, Araña M, López-Martínez T, Moreno C, Merino J, Martino-Rodríguez A, Uixeira A, et al: Treatment of reperfused ischemia with adipose-derived stem cells in a preclinical Swine model of myocardial infarction. Cell Transplant 21: 2723-2733, 2012.

34. Sadat S, Gehmert S, Song YH, Yen Y, Bai X, Gaiser S, Klein H and Alt E: The cardioprotective effect of mesenchymal stem cells is mediated by IGF-I and VEGF. Biochem Biophys Res Commun 363: 674-679, 2007.
35. Tachida Y, Suda K, Nagase H, Shimada K, Isono F and Kobayashi $\mathrm{H}$ : Secreted factors from adipose tissue-derived mesenchymal stem cells suppress oxygen/glucose deprivation-induced cardiomyocyte cell death via furin/PCSK-like enzyme activity. Biochem Biophys Rep 7: 266-272, 2016.

36. Panina YA, Yakimov AS, Komleva YK, Morgun AV,Lopatina OL, Malinovskaya NA, Shuvaev AN, Salmin VV, Taranushenko TE and Salmina AB: Plasticity of adipose tissue-derived stem cells and regulation of angiogenesis. Front Physiol 9: 1656, 2018.

37. Grinnemo KH, Månsson-Broberg A, Leblanc K, Corbascio M, Wärdell E, Siddiqui AJ, Hao X, Sylvén C and Dellgren G: Human mesenchymal stem cells do not differentiate into cardiomyocytes in a cardiac ischemic xenomodel. Ann Med 38: 144-153, 2006.

38. Atsma DE, Fibbe WE and Rabelink TJ: Opportunities and challenges for mesenchymal stem cell-mediated heart repair. Curr Opin Lipidol 18: 645-649, 2007.

39. Tang JM, Wang JN, Zhang L, Zheng F, Yang JY, Kong X, Guo LY, Chen L, Huang YZ, Wan Y and Chen SY: VEGF/SDF-1 promotes cardiac stem cell mobilization and myocardial repair in the infarcted heart. Cardiovasc Res 91: 402-411, 2011.

40. Deuse T, Peter C, Fedak PW, Doyle T, Reichenspurner H, Zimmermann WH, Eschenhagen T, Stein W, Wu JC, Robbins RC and Schrepfer S: Hepatocyte growth factor or vascular endothelial growth factor gene transfer maximizes mesenchymal stem cell-based myocardial salvage after acute myocardial infarction. Circulation 120 (11 Suppl): S247-S254, 2009.

41. Park SG, Kim JH, Xia Y and Sung JH: Generation of reactive oxygen species in adipose-derived stem cells: Friend or foe? Expert Opin Ther Targets 15: 1297-1306, 2011. 